MODELING, IDENTIFICATION AND CONTROL, 1981, vOL. 2, NO. 4, 181-199

doi:10.4173/mic.1981.4.1

\title{
Efficient numerical simulation of ocean hydrodynamics by a splitting procedure
}

\author{
H. BERNTSEN $\dagger$, Z. KOWALIK $\downarrow$, S. SÆLID§ and K. SøRLI $\dagger$
}

Keywords: physical oceanography, numerical modeling, fast and slow modes, splitting, efficiency, accuracy, stability.

A splitting algorithm for fast and slow modes of ocean hydrodynamics is presented. The purpose of the splitting is to reduce the large amount of computational work needed for simulating long real-time periods. The essential point of the splitting is that the external gravity wave terms are extracted from the fully three-dimensional equations of horizontal motion, allowing the reduced equations to be integrated with a larger time step than the original model. The fast external gravity waves are traced by a depth integrated system which is weakly coupled to the reduced three-dimensional momentum equations. The split model shows a radical decrease in computational time and the accuracy is of the same order as in the non-split case.

\section{Introduction}

This paper deals with the development of a mathematical and numerical model of ocean hydrodynamics for computation of velocity fields, temperature fields and salinity fields. The modeling work is a part of the Norwegian research program HAVBIOMODELLER (OCEAN BIO-MODELS) which deals with modeling of the marine ecological system of the Barents Sea. For further description of the program, the reader is referred to Balchen (1979).

A finite difference modeling technique that provides reasonable results in a variety of ocean circulation problems has been documented in the literature (Leendertse et al. (1973), Leendertse and Liu $(1975,1977,1978)$ ). This modeling approach is fully threedimensional, as opposed to the horizontal mean flow modeling in more common use. Vertical structure and exchange mechanisms may be highly significant in some applications, so that the development of three-dimensional modeling techniques represents an important and necessary advance.

One of the major problems related to three-dimensional modeling has been the time consumption of computer simulations. This paper describes a method for reducing the computational time by splitting the horizontal velocity components into two parts. One of the resulting components is the mean depth-integrated value of the horizontal velocity, while the other is the deviation from this mean throughout the depth. The former is mainly governed by the external gravity wave terms. The resulting equation of mean horizontal motion is integrated stepwise with a time step subject to the same restrictive stability condition as in the non-split case. However, the resulting

$\uparrow$ SINTEF, Division of Automatic Control, Trondheim, Norway.

$\ddagger$ Institute of Meteorology and Water Management, Gdynia, Poland.

$\S$ The Norwegian Institute of Technology, Division 'of Engineering Cybernetics, Trondheim, Norway.

Z. Kowalik's work was done while he was a visiting scientist at the Division of Engineering Cybernetics, NTH. 
equation is two-dimensional, which implies much less computing time than in the case of three dimensions.

The remaining three-dimensional equations of velocity deviation throughout the depth involve only internal gravity waves, advective modes and other types of slow modes which are not likely to dominate the stability considerations. Explicit difference schemes of the vertical eddy viscosity terms in the latter equations may introduce a stability limit on the time step which is as restrictive as that caused by the external gravity wave terms. To solve this problem, eddy viscosity terms are treated implicitly in the vertical direction.

This splitting procedure for the horizontal momentum equations reduces the total simulation time radically. The rate of reduction depends on the number of depth layers, complexity of vertical exchange models, etc. However, a rough estimate for a 6-layer model is a reduction of about $95 \%$.

An increase of the time step for the reduced three-dimensional momentum equation implies a linear increase of the time discretization error since the numerical method used is first-order accurate with respect to time. This is of minor importance in our application, since the space discretization errors are likely to be dominant.

Finally it should be mentioned that the idea of splitting itself is not a new one (see Gourlay (1977), Marchuk (1975) and Peaceman and Rachford (1955)), but our approach differs from what is found in the literature. Rather than using splitting as a way of breaking a multidimensional problem down into a sequence of one-dimensional problems (mathematical splitting), this paper describes a splitting of physical modes (physical splitting). The latter approach is also taken by Madala and Piacsek (1977), but their approach deviates entirely from ours in the numerical algorithm used. In $\S \S 2.2$ and 2.3 the algorithm and its implementation is described. In $\S 2.4$ boundary conditions are considered. Chapter three contains simulation results under various conditions. Comparisons between the split and the non-split model are given.

\section{Modeling of physical oceanography}

The matter of interest is the space-time motion of the sea. The following equations are given in a Cartesian coordinate system with the $x$ - and $y$-axes directed to the east and north, respectively, while the positive $z$-axis is oriented upwards. The horizontal plane $z=0$ coincides with the undisturbed sea surface.

\subsection{Basic equations and the non-split model}

In the given coordinate system the equations of horizontal motion for an incompressible source-free, turbulent and rotating fluid are given by the vector equation of evolution

$$
\boldsymbol{v}_{t}+\left[\nabla^{T}\left(\boldsymbol{v} \boldsymbol{v}^{T}\right)\right]^{T}+(w \boldsymbol{v})_{z}=F v-\frac{1}{\rho} \nabla p+a \nabla^{2} v+\frac{1}{\rho} \tau_{z}
$$

where the following notations and symbols are used:

$$
\begin{gathered}
\boldsymbol{v}=[u, v]^{T}-\begin{array}{c}
\text { where } u, v \text { are the velocity components in the } x \text {-and } y \text {-directions, } \\
\text { respectively }
\end{array} \\
w \text { - velocity component in the } z \text {-direction } \\
\boldsymbol{\nabla}=\left[\frac{\partial}{\partial x}, \frac{\partial}{\partial y}\right]^{T}-\text { gradient }\left(\nabla^{T} \nabla=\nabla^{2}\right)
\end{gathered}
$$




$$
\begin{gathered}
F=\left[\begin{array}{cc}
0 & f \\
-f & 0
\end{array}\right] \text { - where } f \text { is the Coriolis parameter } \\
\rho \text { - sea water density } \\
p-\text { pressure } \\
a-\text { horizontal eddy viscosity coefficient } \\
\tau=\left[\tau^{x z}, \tau^{y z}\right]^{T} \text { - where } \tau^{x z}, \tau^{y z} \text { are the vertical stress components in the } x \text { - and } \\
y \text {-directions, respectively }
\end{gathered}
$$

Vertical exchange of momentum is incorporated by the last term of eqn. (2.1). It is given by

$$
\frac{1}{\rho} \tau_{z}=\left(b v_{z}\right)_{z}
$$

where $b$ is the exchange coefficient or the eddy viscosity coefficient. A discussion of the choice of eddy viscosities is included in the Appendix.

Neglecting the acceleration in the vertical momentum equation this equation reduces to the hydrostatic equation

$$
p_{z}=-\rho g
$$

The ocean fluid may be considered as incompressible which implies that the following continuity equation is valid

$$
\nabla^{T} v+w_{z}=0
$$

The space-time variations of water density, $\rho$, are modelled by a functional relationship

$$
\rho=\rho(S, T)
$$

where $S$ and $T$ are the salinity and the temperature of the water, respectively. These scalar fields are modelled by a balance equation in the form

$$
c_{t}+\nabla^{T}(v c)+(w c)_{z}=K_{H} \nabla^{2} c+\left(K_{V} c_{z}\right)_{z}, \quad c=S, T
$$

where $K_{H}$ and $K_{V}$ are the horizontal and the vertical mass (thermal) diffusion coefficients.

For numerical solution of the eqns. (2.1)-(2.5), the flow region is partitioned into a finite number of boxes. A vertical intersection is shown in Fig. 1. The staggered distribution of the dependent variables in a general box is illustrated in Fig. 2.

The finite difference approximations of the eqns. (2.1)-(2.5) can be divided into three categories:

(i) derivation of the layer-integrated equations

(ii) approximation of space derivatives of the layer equations

(iii) approximation of time derivatives giving a time-stepping procedure 


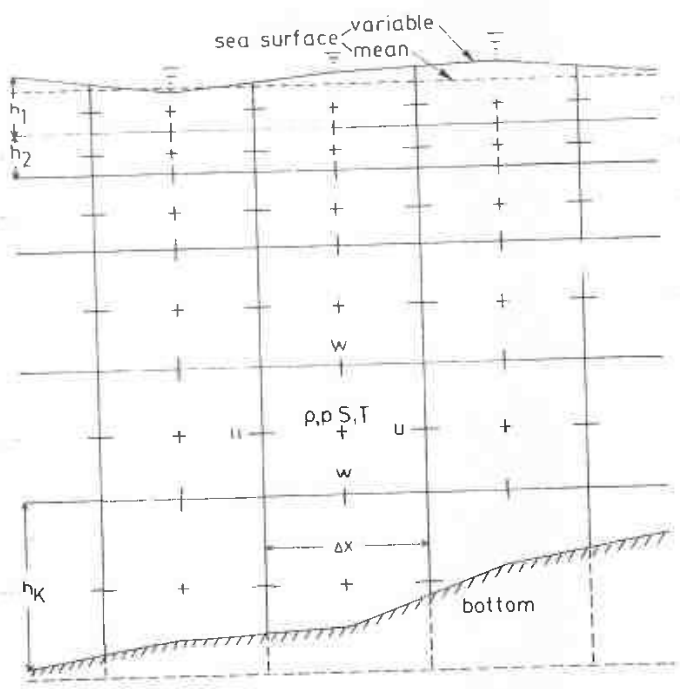

Figure 1. Vertical section of grid points. The layers may have different thicknesses. The yer thickness varies in the horizontal plane in accordance with the actual depths of the ocean. The top layer thickness varies with space and time due to the rise and fall of the sea surface.

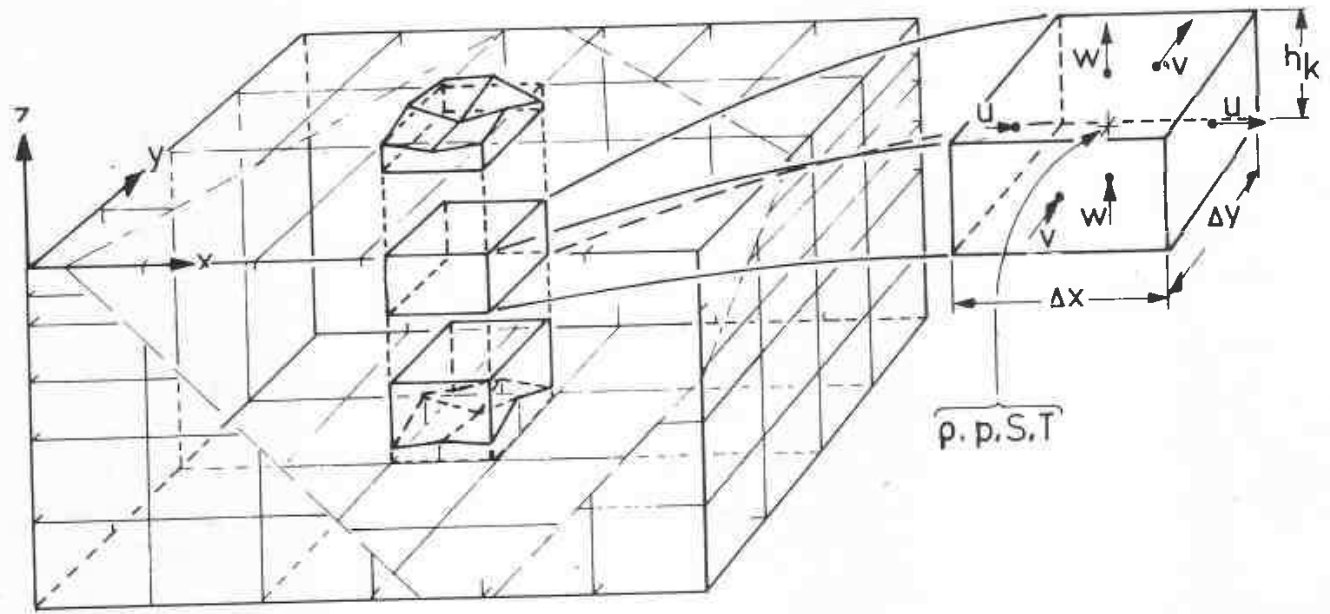

Figure 2. A three-dimensional distribution of grid points. The locations of the velocity components are indicated on the extracted box. The remaining model variables are associated with the centre points of the boxes.

The non-split scheme is quite identical to the one used by Leendertse et al. (1973). The order of calculation is as follows:

(i) compute pressure gradient terms at level $t_{n}$

(ii) compute vertical velocity at $t_{n}$

(iii) compute elevation at $t_{n+1}$

(iv) compute temperature and salinity at $t_{n+1}$

(v) compute density at $t_{n+1}$

(vi) compute horizontal velocities at $t_{n+1}$ 
For the horizontal velocity an explicit leap-frog time-stepping scheme in the form

$$
v^{n+1}=v^{n-1}+2 \Delta t D^{n, n-1}
$$

is used, where $\boldsymbol{D}$ contain all terms contributing to a time rate of change of $\boldsymbol{v}$. A similar procedure is used for both salinity and temperature. For further details the reader is referred to Leendertse et al. (1973).

\subsection{Derivation and analysis of the split model}

As mentioned previously, the non-split model is very time-consuming due to the rather restrictive stability condition,

$$
\Delta t \leqslant \frac{\Delta l}{\sqrt{ }(2 g H)}, \Delta l=\min \{\Delta x, \Delta y\}
$$

associated with the given method for the linearized model. In order to improve on this severe condition for the full three-dimensional model, the external gravity wave terms are extracted from the $3 D$-model $\dagger$ and traced by a depth-integrated $2 D$-model. In this way a larger time step may be used in the reduced $3 D$-model. The $2 D$-model, however, must be integrated using a small time step restricted by inequality (2.9). The overall procedure is then likely to be much more efficient than the non-split procedure. The larger the number of layers, the larger is the reduction in computing time. The timestepping of the two models is illustrated in Fig. 3.

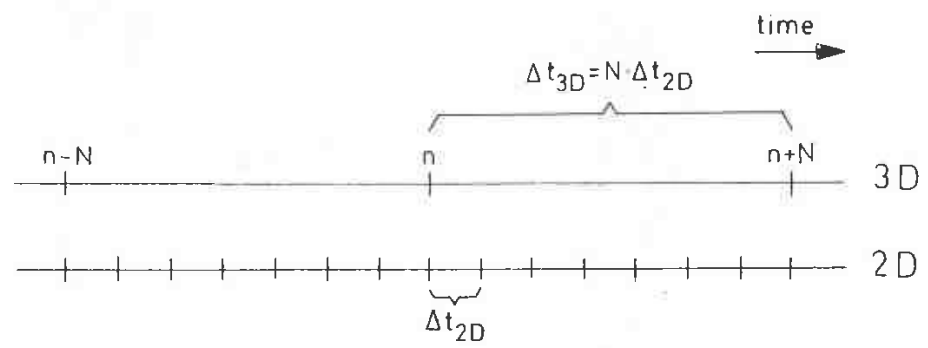

Figure 3. Time-stepping of the split equations. The three-dimensional $(3 D)$ model of slow mode computations operates with a time-step $\Delta t_{3 D}=t_{n+N}-t_{n}$, while the twodimensional $(2 D)$ model of fast mode computations operates with a time-step $\Delta t_{2 D}=t_{n+1}-t_{n}$, i.e. $\Delta t_{3 D}=N \Delta t_{2 D}$. A typical value of $N$ is 30 .

The splitting is produced by decomposing the horizontal vector $v$ into one vertically averaged value, $\boldsymbol{V}$, and a deviation from that mean value, $\hat{\boldsymbol{v}}$

$$
\boldsymbol{v}=\boldsymbol{V}+\widehat{\boldsymbol{v}}, \boldsymbol{V}=\frac{1}{H} \int_{-h}^{\zeta} \boldsymbol{v} d z
$$

where $\zeta$ is the water elevation relative to the reference plane $z=0, h$ is the distance from this plane to the bottom and $H=\zeta+h$. Furthermore, the hydrostatic equation (2.2) is integrated to give the pressure

$$
p(z)=p_{s}+g \int_{z}^{\zeta} \rho(\sigma) d \sigma
$$

$\uparrow$ Three-dimensional model. 
where $p_{S}$ is the atmospheric pressure at the sea surface. Hence,

$$
\frac{1}{\rho} \nabla p=\frac{1}{\rho} \nabla p_{S}+g \nabla \zeta+\frac{1}{\rho} \widehat{\nabla} p
$$

where

$$
\widehat{\nabla} p=g \nabla \int_{z}^{\zeta} \hat{\rho} d \sigma-g \hat{\rho} \nabla \zeta, \hat{\rho}=\rho-\rho_{0}
$$

The density variations, given by $\hat{\rho}$, are usually much less than the constant density parameter $\rho_{0}$. Substituting eqn. (2.11) into eqn. (2.1) and integrating over depth yields

$$
V_{t}=F V-g \nabla \zeta+a \nabla^{2} V+\frac{1}{H}\left[\left(\frac{1}{\rho} \tau\right)_{S}-\left(\frac{1}{\rho} \tau\right)_{B}\right]+A
$$

where $\tau_{S}$ is the wind stress vector and $\tau_{B}$ is the vector of bottom friction. These expressions are determined by the boundary conditions at the sea surface and the bottom as considered in $\S 2.4$. $A$ is given by

$$
A=\frac{1}{H} \int_{-h}^{\zeta}\left[-\left[\nabla^{T}\left(v v^{T}\right)\right]^{T}-(w v)_{z}-\frac{1}{\rho} \widehat{\nabla} p+a \nabla^{2} \hat{v}\right] d z
$$

Depth-integrating the continuity equation, (2.3), yields

$$
\zeta_{t}=-\nabla^{T}(H V)
$$

Multiplying eqn. (2.13a) by $H$ and substituting eqn. (2.15) give

$$
(H V)_{t}+\left(\nabla^{T}(H V)\right) V=F H V-g H \nabla \zeta+a H \nabla^{2} V+\left(\frac{1}{\rho} \tau\right)_{S}-\left(\frac{1}{\rho} \tau\right)_{B}+H A
$$

which is another useful form of the depth-integrated horizontal momentum equation. Equations (2.13) and (2.15) constitute the two-dimensional model of vertically averaged horizontal motion of water and sea surface elevation. The source term $A$ is determined by the three-dimensional model described in the following. Subtracting eqn. (2.13) from eqn. (2.1) gives

$$
\begin{aligned}
\hat{v}_{t}+\left[\nabla^{T}\left(v v^{T}\right)\right]^{T}+(w v)_{z}=F \hat{v}-\frac{1}{\rho} \widehat{\nabla} p+a \nabla^{2} \hat{v} & +\frac{1}{\rho} \tau_{z} \\
& -\frac{1}{H}\left[\left(\frac{1}{\rho} \tau\right)_{S}-\left(\frac{1}{\rho} \tau\right)_{B}\right]-A
\end{aligned}
$$

The last equation has almost the same form as the non-split momentum equation (2.1) except for the advection terms, which involve non-split velocities, and the last two terms on the right hand side, which involve the depth-integral of advection terms, pressure terms and eddy diffusion terms. In eqn. (2.16) the term governing the fast moving external gravity waves has been eliminated. By using the mean value theorem for integrals, eqn. (2.12) may be written as

$$
\widehat{\nabla}=\left(\hat{\rho}_{*}-\hat{\rho}\right) g \nabla \zeta+g(\zeta-z) \nabla \hat{\rho}_{*}
$$

where $\hat{\rho}_{*}=\hat{\rho}\left(x, y, z_{*}\right)$ for $-h \leqslant z_{*} \leqslant \zeta$. The slowly moving internal gravity waves caused by water density variations are governed by the terms of eqn. (2.17). The important term with respect to stability requirements of a given numerical method is 
the first term on the right-hand side of the equation. For almost all explicit methods this will be of the form

$$
\Delta t \leqslant \frac{\Delta l}{\sqrt{ }(2 g H)} \sqrt{ }\left(\frac{\rho}{\left|\hat{\rho}_{*}-\hat{\rho}\right|}\right)
$$

which is much less restrictive than the condition (2.9) for the non-split model. For instance, if $\rho /\left|\hat{\rho}_{*}-\hat{\rho}\right|$ is of order $10^{4}$, which is a reasonable value, the upper bound of inequality (2.18) is 100 times larger than that of inequality (2.9). However, numerical solution of eqn. (2.16) by explicit methods is not likely to be affected by stability conditions attached to the pressure term $(1 / \rho) \widehat{\nabla p}$. The vertical exchange of momentum, governed by the term $(1 / \rho) \tau_{z}$, is most often the critical one. A stability analysis of a linearized pure eddy diffusion problem, solved by the explicit Euler method, gives the following stability limitation

$$
\Delta t \leqslant \frac{(\Delta z)^{2}}{4 b}, \Delta z=\min _{k}\left\{h_{k}\right\}
$$

where $b$ is the vertical eddy diffusion coefficient. This may in some cases cause limitations on the time step that are too restrictive. Due to this fact an implicit treatment of the vertical eddy diffusion terms is needed in order to eliminate the stability constraints imposed by eqn. (2.19).

\subsection{Numerical solution and implementation of the split model}

In the split model the same order of calculation with respect to the dependent variables is used as in the non-split model. The finite difference approximations are also of the same kind, i.e. central differences replacing space and time derivatives. For eqn. (2.16) this results in the layer equations

$$
\begin{aligned}
&\left(h_{k} \hat{v}_{k}\right)_{i}+\left[\nabla^{T}\left(h_{k} v_{k} v_{k}^{T}\right)\right]^{T}+\delta_{z}\left(w_{k} v_{k}\right)=F h_{k} \hat{v}_{k}-\frac{h_{k}}{\rho_{k}} \widehat{\nabla}_{p_{k}}+a \nabla^{2}\left(h_{k} \hat{v}_{k}\right) \\
&+\delta_{z}\left(\frac{1}{\rho} \tau\right)_{k}-\frac{h_{k}}{H}\left[\left(\frac{1}{\rho} \tau\right)_{S}-\left(\frac{1}{\rho} \tau\right)_{B}\right]-h_{k} A
\end{aligned}
$$

where $\tau_{S}$ is the wind stress, $\tau_{B}$ is the bottom friction and $\tau_{k+\frac{1}{2}}$ is the momentum exchange between layer number $k$ and number $k+1$. Furthermore, $\delta_{z}$ denotes the central difference operator. Neglecting the approximations of the horizontal space derivatives, the following numerical scheme is used

$$
\begin{aligned}
\left(h_{k} \hat{v}_{k}\right)^{n+N}=\left(h_{k} \hat{v}_{k}\right)^{n-N} & +2 N \Delta t\left[-\nabla^{T}\left(h_{k} v_{k} v_{k}^{T}\right)^{n}-\delta_{z}\left(w_{k} v_{k}\right)^{n}\right. \\
& +F\left(h_{k} \hat{v}_{k}\right)^{n}-\left(\frac{h_{k}}{\rho_{k}} \widehat{\nabla}_{p_{k}}\right)^{n}+a \nabla^{2}\left(h_{k} \hat{v}_{k}\right)^{n-N} \\
+ & \left.\delta_{z}\left(\frac{1}{\rho_{k}{ }^{n}} \tau_{k}^{n+N}\right)-\left(\frac{h_{k}}{H}\right)^{n}\left\{\left(\frac{1}{\rho} \tau\right)_{S}^{n+N}-\left(\frac{1}{\rho} \tau\right)_{B}^{n+N}\right\}-\left(h_{k} A\right)^{n+N}\right]
\end{aligned}
$$

for all layers, $k=1(1) K$, where $K$ is the local number of layers. Having defined the three-level scheme of eqn. (2.21), two main problems are encountered. First, the approximation of the integral term $A^{n+N}$ given by eqn. (2.14) should be accurate and efficiently implemented. Second, the implicit terms of eqn. (2.21) give origin to a system 
of linear, algebraic equations that must be solved for each water column. Solutions to these problems are given in the algorithm below. The determination of $A^{n+N}$ is based on definition (2.10), which implies

$$
\int_{-h}^{\zeta} \hat{v} d z=0, t>0
$$

which has the discrete counterpart

$$
\sum_{k=1}^{K}\left(h_{k} \hat{v}_{k}\right)^{n}=0, n>0
$$

Summing eqn. (2.21) over $k$ and using eqn. (2.22), gives

$$
(H A)^{n+N}=\sum_{k=1}^{K}\left[-\nabla\left(h_{k} \boldsymbol{v}_{k}^{T} \boldsymbol{v}_{k}\right)^{n}-\delta_{z}\left(w_{k} \boldsymbol{v}_{k}\right)^{n}-\left(\frac{h_{k}}{\rho_{k}} \widehat{\nabla} p_{k}\right)^{n}\right]
$$

The numerical algorithm for the split model of horizontal momentum is summarized in the following:

\section{Algorithm}

For all water columns of the $3 D$-model,

(i)

$$
\begin{aligned}
w_{K+\frac{1}{2}}{ }^{n} & =0 \\
w_{k-\frac{1}{2}}{ }^{n} & =w_{k+\frac{1}{2}}{ }^{n}-\nabla^{T}\left(h_{k} v_{k}\right)^{n}, k=K(-1) 1 \\
\zeta^{n+N} & =\zeta^{n-N}+2 N \Delta t \cdot w_{\frac{1}{2}}^{n}
\end{aligned}
$$

which gives preliminary elevations at $t_{n+N}$.

(ii)

$$
\left(h_{k} \hat{v}_{k}^{*}\right)^{n+N}=\left(h_{k} \hat{v}_{k}\right)^{n-N}+2 N \Delta t E^{n, n-N}, k=K(-1) 1
$$

where $E$ contains all the explicit terms within the brackets of eqn. (2.21). This gives preliminary velocities, $\hat{v}_{k}$, at the time-level $t_{n+N}$.

$$
(H \boldsymbol{A})^{n+N}=\frac{1}{2 N \Delta t} \sum_{k=1}^{K}\left(h_{k} \hat{\boldsymbol{v}}_{k}^{*}\right)^{n+N}
$$

which is accumulated from step (ii). This defines $A^{n+N}$.

(iv) $\left(h_{k} \widehat{v}_{k}\right)^{n+N}=\left(h_{k} \hat{v}_{k}^{*}\right)^{n+N}+2 N \Delta t\left\{\delta_{z}\left(\frac{1}{\rho_{k}{ }^{n}} \tau_{k}^{n+N}\right)-\left(\frac{h_{k}}{H}\right)^{n}\left[\left(\frac{1}{\rho}-\tau\right)_{S}^{n+N}\right.\right.$

$$
\left.\left.-\left(\frac{1}{\rho} \tau\right)_{B}^{n+N}\right]-\left(h_{k} A\right)^{n+N}\right\}
$$

for $k=1(1) K$, which constitute a linear system of equations for $\hat{v}_{1}{ }^{n+N}, \ldots$, $\hat{v}_{K}{ }^{n+N}$, since $\tau_{k}{ }^{n+N}$ is given by

$$
\tau_{k}{ }^{n+N}=\rho_{k}{ }^{n} b_{k}{ }^{n} \frac{\delta_{z} v_{k}{ }^{n+N}}{h_{k}{ }^{n+N}}
$$

and $\tau_{S}{ }^{n+N}=\tau_{\frac{1}{2}}{ }^{n+N}$ and $\tau_{B}{ }^{n+N}=\tau_{K+\frac{1}{2}}{ }^{n+N}$, which, are the surface wind-induced stress and bottom current-induced drag, respectively. The latter conditions are discussed in $\S 2.4$. Here it is noted that when $\tau_{B}{ }^{n+N}$ does not depend on $v_{K}{ }^{n+N}$, a tridiagonal system of equations results which is easily solved because 
of its simplicity and its diagonal dominance. For implicit bottom stress computations the reader is referred to $\S 2.4$. Continuing with the depthintegrated $2 D$-model, for $m=n(1)(N+n-1)$ :

$$
\zeta^{m+1}=\zeta^{m-1}-2 \Delta t \nabla^{T}(H V)^{m}
$$

(vi) $H^{m+1} V^{m+1}=H^{m-1} V^{m-1}+2 \Delta t\left[F H^{m} V^{m}-g H^{m} \nabla \zeta^{m}-a H^{m-1} \nabla^{2} V^{m-1}\right.$

$$
\left.+\left(\frac{1}{\rho} \tau_{\mathrm{S}}\right)^{m+1}-\left(\frac{1}{\rho} \tau_{B}\right)^{m+1}+H^{m} A^{n+N}\right]
$$

where $A^{n+N}$ is given from (iii). This gives the total horizontal velocity

$$
v_{k}^{n+N}=V^{n+N}+\hat{\boldsymbol{v}}_{k}^{n+N}, k=1(1) K
$$

Note that the depth-integrated model involves a source-like term $A^{n+N}$ evaluated by the $3 D$-model. A suggestion is that $A^{n}$ and $A^{n+N}$ should be used to produce linear interpolation values of the integral term at the equidistant interpolation points $t_{n+1}, t_{n+2}, \ldots, t_{n+N}$ and replace the discontinuous source term by a continuous one in the depth-integrated model.

Temperature and salinity fields, given by eqn. (2.5), are calculated in the same way as in the non-split model. The only difference is that vertical exchange of heat and salt are modelled implicitly because of the larger time step that is used in these calculations.

\subsection{Boundary conditions}

The wind stress is given as a function of the wind velocity $\boldsymbol{w}_{\boldsymbol{a}}$

$$
\boldsymbol{\tau}_{s}=v \rho_{a}\left|\boldsymbol{w}_{a}\right| \boldsymbol{w}_{a}
$$

where $\rho_{a}$ is the air density and $v$ is some proportionality constant. The bottom stress term is also given by a quadratic law

$$
\tau_{B}=C \rho_{B}\left|\boldsymbol{v}_{K}\right| \boldsymbol{v}_{K}
$$

where $C$ is Chezy's coefficient and $\rho_{B}$ is the water density in the bottom layer. The wind stress of eqn. (2.25) is easily implemented into the implicit treatment of vertical momentum exchange. The condition of the bottom friction is more difficult to handle implicitly in the split model. The reason for this difficulty is twofold: (i) it is a nonlinear condition, (ii) the depth-integrated velocity $V$ is not known at the advanced time-level $t_{n+N}$. The condition is

$$
\left(\frac{1}{\rho} \tau\right)_{B}=C\left|v_{K}\right| v_{K}=C\left|\hat{v}_{K}+V\right|\left(\hat{v}_{K}+V\right)
$$

An explicit difference replacement of the last equation is

$$
\left(\frac{1}{\rho} \tau\right)_{B}^{n+N}=C\left|v_{K}{ }^{n}\right| v_{K}{ }^{n}
$$

which implies that the equation of step (iv) of the algorithm defines two tridiagonal systems of equations for each water column (one system for each horizontal direction). 
Simulations indicate that this approximation produces false oscillations starting from the bottom. Instead a quasi-implicit replacement of eqn. (2.26) is used:

$$
\left(\frac{1}{\rho} \tau\right)_{B}^{n+N}=C\left|\boldsymbol{v}_{K}{ }^{n-N}\right|\left(\hat{\boldsymbol{v}}_{K}{ }^{n+N}+\boldsymbol{V}^{n-N}\right)
$$

In this case the system of equations is no longer tridiagonal. In addition to the three diagonals the coefficient matrices contain non-vanishing elements in the last column. The latter approximation is much more stable than the approximation of eqn. (2.27). The bottom stress condition of the depth-integrated model is handled in a similar way by

$$
\left(\frac{1}{\rho} \tau\right)_{B}^{n+1}=C\left|\boldsymbol{v}_{K}^{n-N}\right|\left(\hat{\boldsymbol{v}}_{K}{ }^{n+N}+V^{m-1}\right)
$$

\section{Simulations}

In the first section of this chapter a comparison of the non-split model and the split model is done. The main object is to examine the computational efficiency of the split procedure versus the non-split one. The results are presented in the Table. Another important question is whether or not the split procedure gives numerical approximations of the same order of accuracy as the non-split procedure. The answer is more of less given in Figs. 6-7 and comments on this are given below.

\begin{tabular}{ccccc}
\hline $\begin{array}{c}\text { Slow mode } \\
\text { time step } \\
\text { (minutes) }\end{array}$ & $\begin{array}{c}\text { Computing } \\
\text { time } \\
\text { (minutes) }\end{array}$ & $\begin{array}{c}\text { Reduction } \\
(\%)\end{array}$ & $\begin{array}{c}\text { Computing time/ } \\
\text { real time }\end{array}$ & $\begin{array}{c}\text { Non-split/split } \\
\text { time ratio }\end{array}$ \\
\hline 2 & $25 \cdot 38$ & 0 & $1 / 13$ & $1 \cdot 0$ \\
40 & $2 \cdot 16$ & 91.4 & $1 / 153$ & 11.8 \\
80 & 1.59 & 93.7 & $1 / 208$ & 16.0 \\
160 & 1.36 & 94.6 & $1 / 242$ & 18.6 \\
\hline
\end{tabular}

Comparison of computational efficiency for problem B using different slow mode time steps. (Fast mode time step is 2 min.)

As mentioned before, only simulations on a closed boundary domain are presented in this paper. However, in a later report open sea boundary conditions will be introduced into the existing model. To test the computational code of the split model, several test cases have been investigated. Simulation tests of variable complexity both with respect to boundary geometry and driving forces have been performed. The driving forces were mainly wind stress at the sea surface and the initial salinity distribution. In $\S 3.2$ (Figs. 9-12), results simulating wind and density driven currents are shown. These demonstrate the model's ability to produce physically reasonable simulations.

\subsection{Comparison of the split and the non-split model}

Two problems for numerical tests of the efficiency and the accuracy of the split procedure were considered. 


\section{Problem A}

This simulation is designed in order to test the accuracy of the split model as compared to the non-split model. The problem is defined on a two-dimensional vertical section of an oceanic domain. The numerical grid is arranged in a $24 \times 6$ grid as shown in Fig. 4. The grid size is $25 \mathrm{~m}$ in the vertical $z$-direction and $40 \mathrm{~km}$ in the horizontal $x$-direction. The initial conditions are given by zero velocity throughout the basin, an initial salinity distribution as shown in Fig. 5, and a level sea surface. The

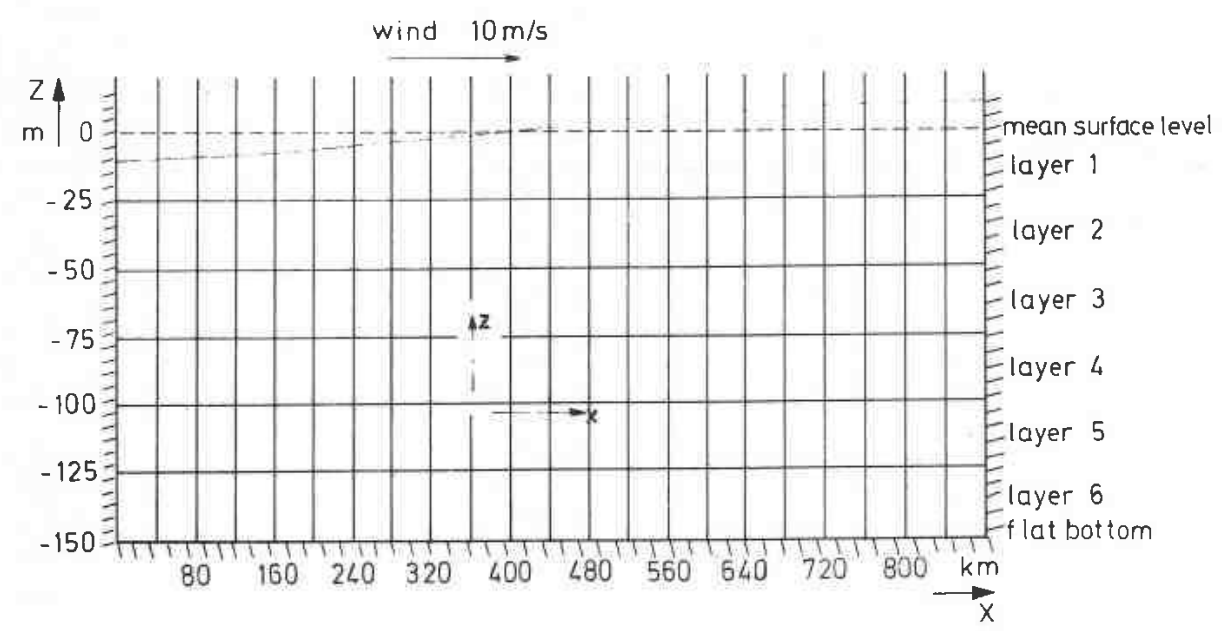

Figure 4. The rectangular flow region of Problem A. The flat-bottomed basin was divided into 6 depth layers of constant thickness $25 \mathrm{~m}$.

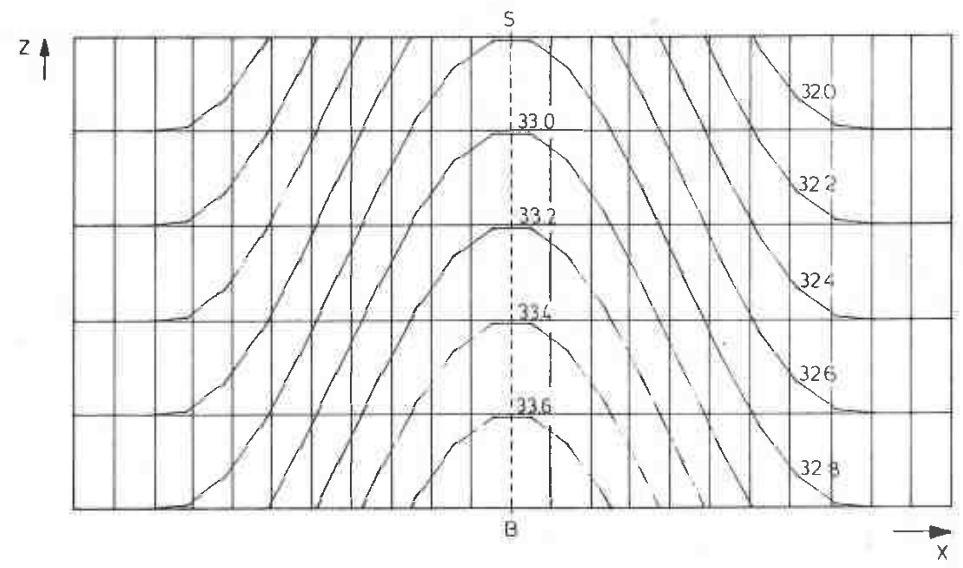

Figure 5. Initial salinity profile of Problems $A$ and $B\left[\mathrm{~kg} / \mathrm{m}^{3}\right]$. The initial distribution of salinity of the three-dimensional problem is obtained by rotating the vertical profile of the figure about the vertical axis $S B$. 
system is driven by a wind stress generated from an easterly wind velocity of $10 \mathrm{~m} / \mathrm{s}$ along the sea surface. The wind stress will set up an easterly current in the positive $x$-direction in the surface layer, and a westerly current in the bottom layers. The model is run for a $13 \mathrm{~h}$ period of real time, and some results are shown in Figs. 6 and 7. The currents of the slow mode part of the model at $x=480 \mathrm{~km}$ and at different depth layers are shown in Fig. 6 for different values of $N$. As expected, we observe that the

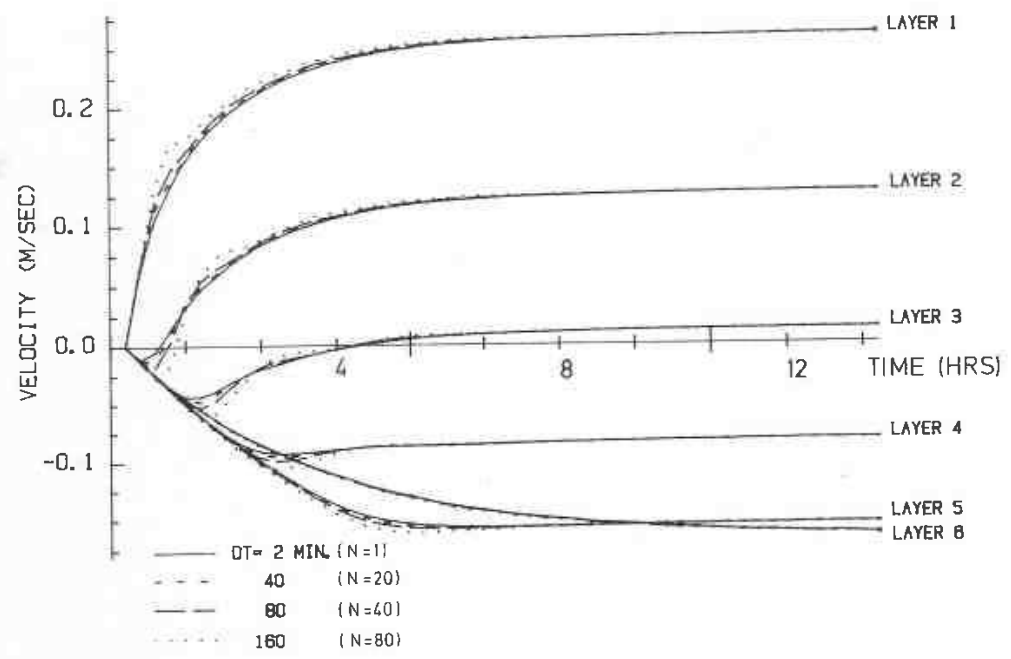

Figure 6. Slow mode layer velocities along the central vertical line as functions of time (Problem A). After about five hours there is a nearly stationary horizontal flow field in all layers except the bottom, which needs a little more time to approach stationarity. The non-split $(D T=2 \mathrm{~min})$ and the split procedure $(D T=40,80,160 \mathrm{~min})$ produce results that differ slightly in the transient period, due to different discretization errors.

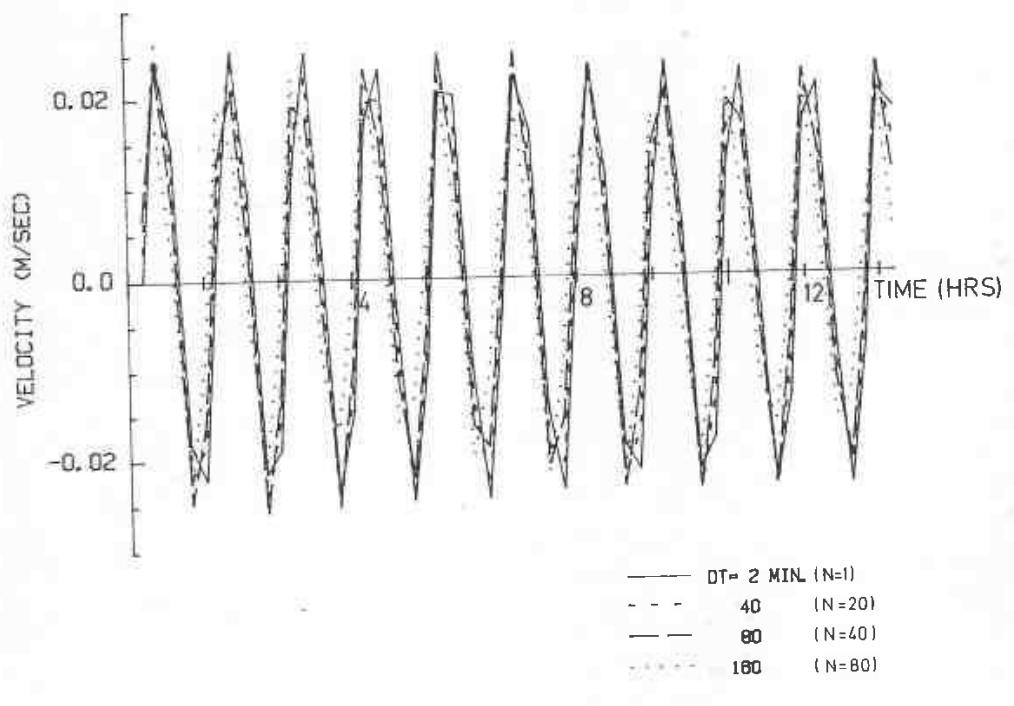

Figure 7. Fast mode depth-integrated velocities at the centre point as functions of time (Problem A). The four curves of this figure are produced together with the corresponding curves of the layer velocities of Fig. 6. 
deviation between the split model $(N>1)$ and the non-split model $(N=1)$ increases as $N$ increases. The steady state deviation is, however, zero. During the transient period the deviation between the split and the non-split model is sufficiently small except, may be, for the case where $N=80\left(\Delta t_{3 D}=160 \mathrm{~min}\right)$. Figure 7 shows the fast mode velocities for the same values of $N$. Again we observe no significant variation between the non-split and the split simulations. As a conclusion we may say that a slow mode time step of $80 \mathrm{~min}$ seems to be quite acceptable.

As a final note on accuracy, we should mention that the approximation of the non-linear bottom stress condition of eqn. (2.26) for the split model may be critical to the overall procedure. Although the pure implicit treatment of the vertical exchange of momentum is an unconditionally stable procedure internally, the bottom stress approximation of eqn. (2.28) may cause the total procedure to be only conditionally stable. Further analysis of this problem is needed. Presently a moderate slow-mode time step less than $90 \mathrm{~min}$ is used, in order to eliminate growing instabilities from the non-linear condition at the bottom.

\section{Problem B}

Problem B is designed to test the computational time efficiency of the splitting procedure when used on a general 3-dimensional closed domain. As an example the bottom topography of the Barents Sea is used as a model domain (see Fig. 8). The open boundaries are artificially closed in order to simplify the computations. Results from more realistic simulations using open boundaries will be published later.

The grid used is a $24 \times 24$ grid in the horizontal plane and 6 layers of variable thickness in the vertical direction $(25 \mathrm{~m}, 25 \mathrm{~m}, 50 \mathrm{~m}, 100 \mathrm{~m}, 100 \mathrm{~m}, 200 \mathrm{~m})$. The horizontal grid size is $40 \mathrm{~km}$.

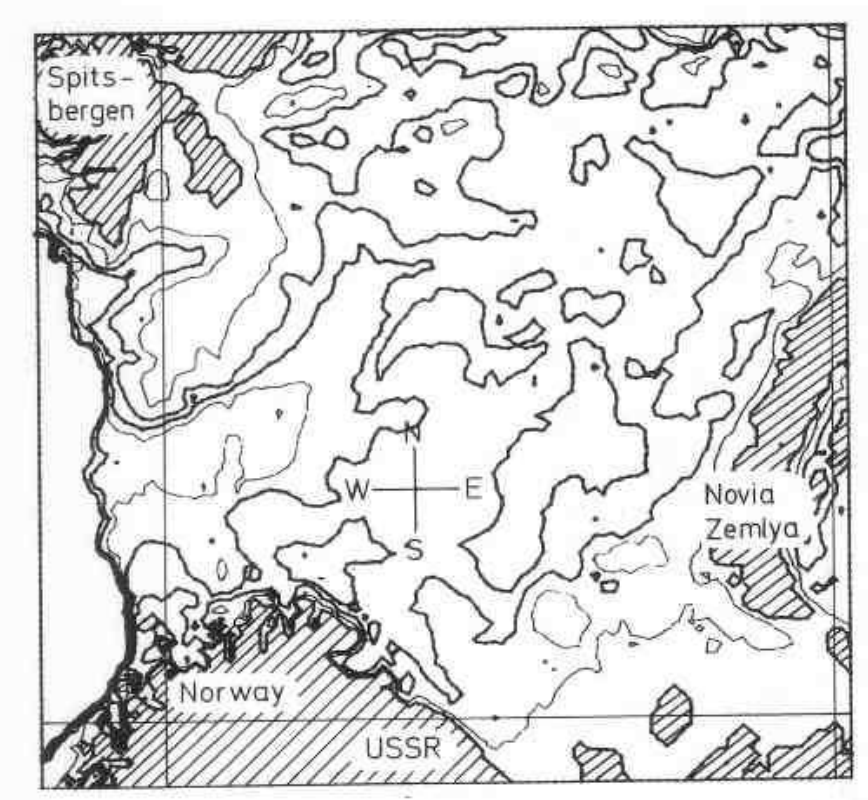

Figure 8. Depth map of the Barents Sea. Distance between isolines is $100 \mathrm{~m}$. The flow region of Problem B was the Barents Sea with closed boundaries. The layer thickness of the 6-layer model were $25,25,50,100,100$ and $200 \mathrm{~m}$ from top to bottom. 
The initial velocity field is set to zero and the initial sea level is horizontal. The initial salinity is given as a symmetric distribution around the vertical axis at $(x, y)=$ $(480 \mathrm{~km}, 480 \mathrm{~km})$. A vertical cut through this axis yields the same salinity field as shown in Fig. 5.

The external driving force is a south-westerly wind blowing with a strength of $10 \mathrm{~m} / \mathrm{s}$. Some of the simulation results are shown in Figs. 9 to 12. Figures 9 and 11 show the total horizontal velocity field for different times and for different depths. The velocity vectors point outwards from the grid points (indicated by dots in the figures), and the strength of the current is indicated by the velocity scale given below

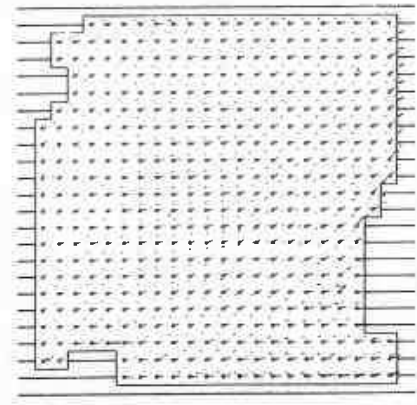

(a) $t=2$ hours

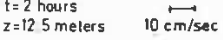

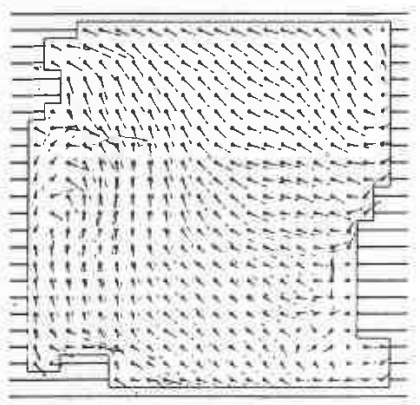

(b) $1=10$ hours $I=10$ hours
$z=12.5$ meter

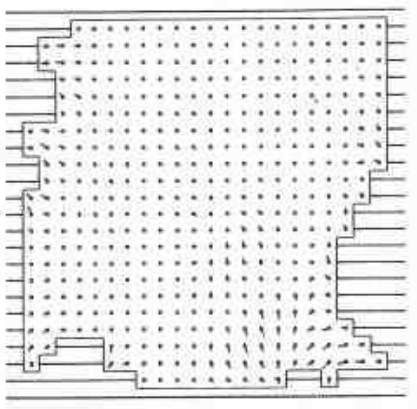

(a) $\begin{aligned} & t=2 \text { hours } \\ & z=75 \text { meters }\end{aligned}$

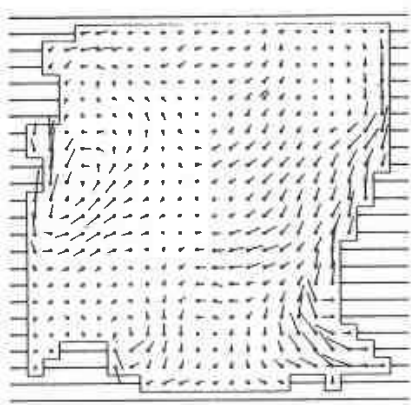

(b) $\begin{aligned} & \boldsymbol{x}=10 \text { hours } \\ & \mathrm{z}=75 \text { meters }\end{aligned} \quad 10 \mathrm{~cm} / \mathrm{sec}$

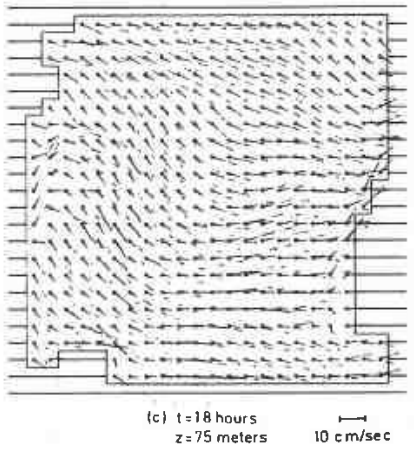

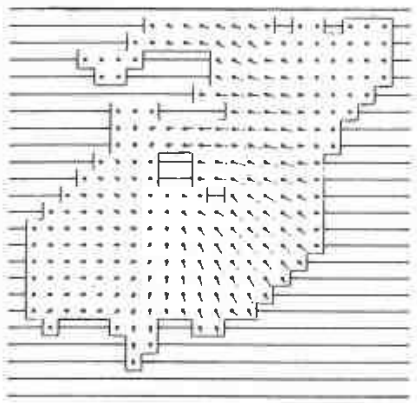

(a) $\begin{aligned} & t=2 \text { hours } \\ & z=250 \text { meters }\end{aligned} 10 \stackrel{\mathrm{cm} / \mathrm{sec}}{\longrightarrow}$

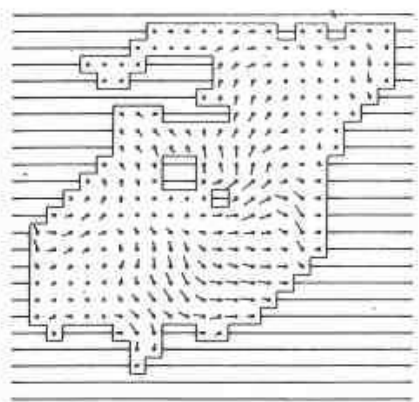

(b) $\begin{aligned} & 2=10 \text { hours } \\ & z=250 \text { melers }\end{aligned}$

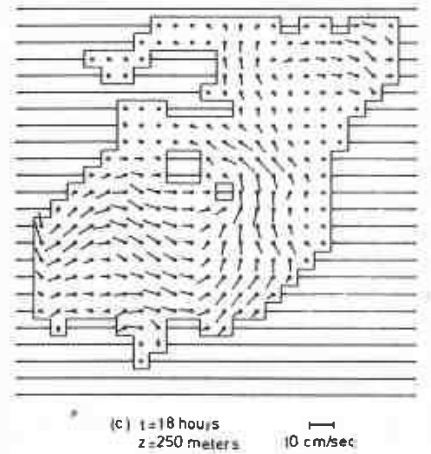

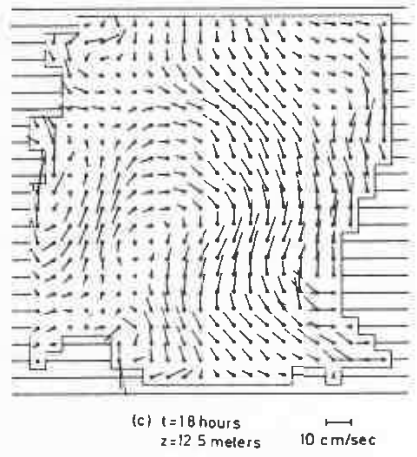

Figure 9. Horizontal velocity fields for times 2,10 and $18 \mathrm{~h}$, and depths $12 \cdot 5,75$ and $250 \mathrm{~m}$. 


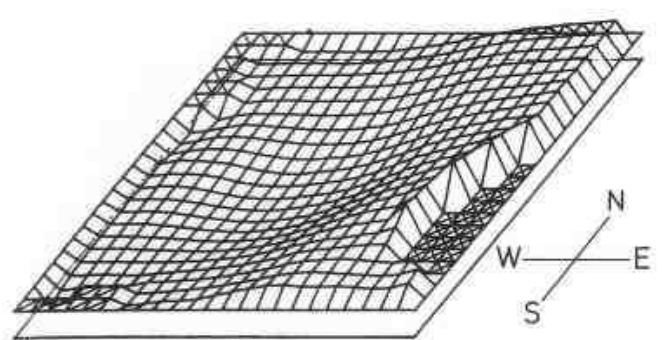

(a) $t=2$ hours

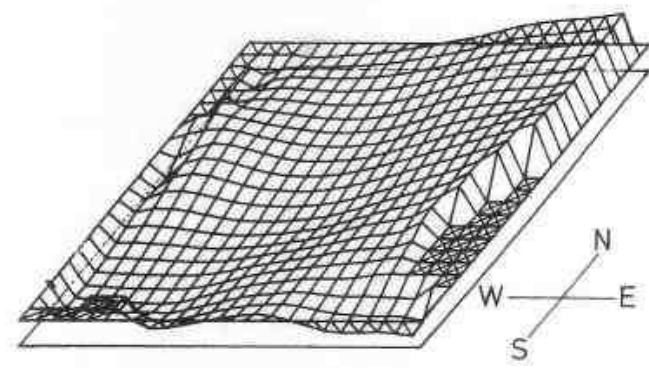

(b) $\mathrm{t}=10$ hours

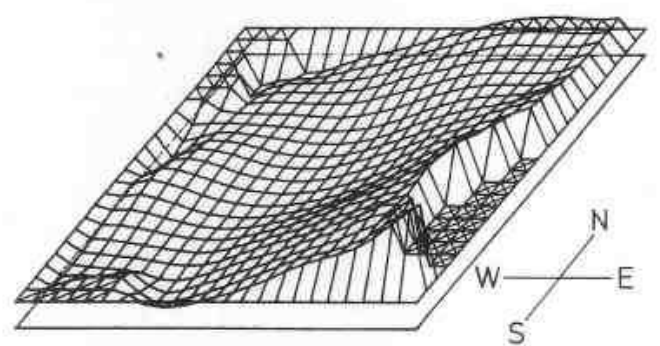

(c) $\hat{\imath}=18$ hours

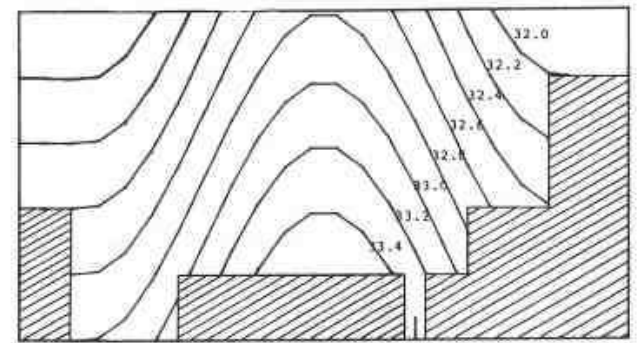

(a) $t=2$ hours

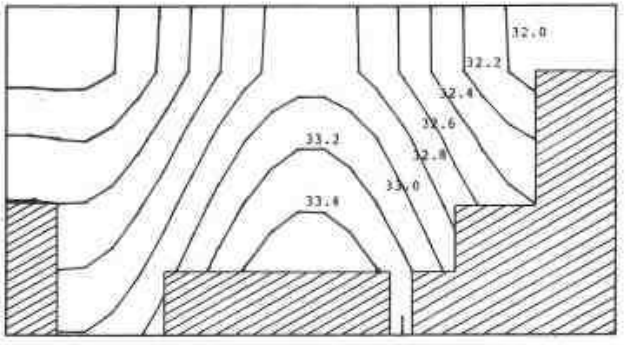

(b) $t=10$ hours

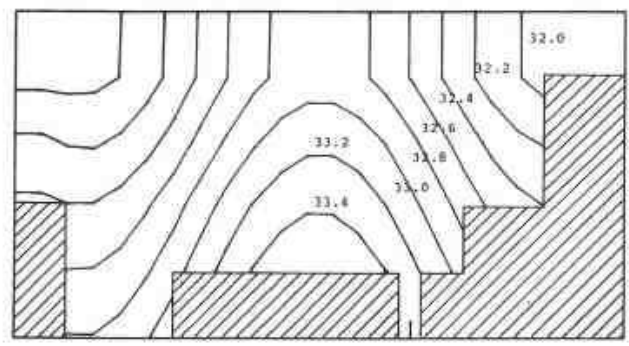

(c) $t=18$ hours

Figure 10. Left: Sea surface elevation plot for times 2, 10 and $18 \mathrm{~h}$. The elevation vary between $20 \mathrm{~cm}$ below and $20 \mathrm{~cm}$ above the mean sea surface level. The land areas of Novaya Zemlya are marked by + , the northern parts of Norway by and Spitzbergen by $\backslash$. Right: Salinity distributions for the east/west section located at $y=480 \mathrm{~km}$ for the times 2, 10 and $18 \mathrm{~h}$. Note that each layer is represented by the same thickness in the figure.

each velocity plot. We observe the combined effect of the current fields of the surface wind stress, the density gradients, the Coriolis force and the boundary geometry including the free surface. The free surface elevation is shown at different times at the $3 D$-plots to the left in Figs. 10 and 12.

Considering the current development at $z=12.5 \mathrm{~m}$, as shown in Figs. 9 and 11 , it is seen that the surface drift current is predominantly to the right of the wind direction due to the Coriolis effect, except along the southern and the eastern boundaries where a belt of longshore drift is established. Near the middle of the ocean the surface drift current is also influenced by the initial salinity distribution, giving rise to an eddy pattern in the final stage of the simulation. 

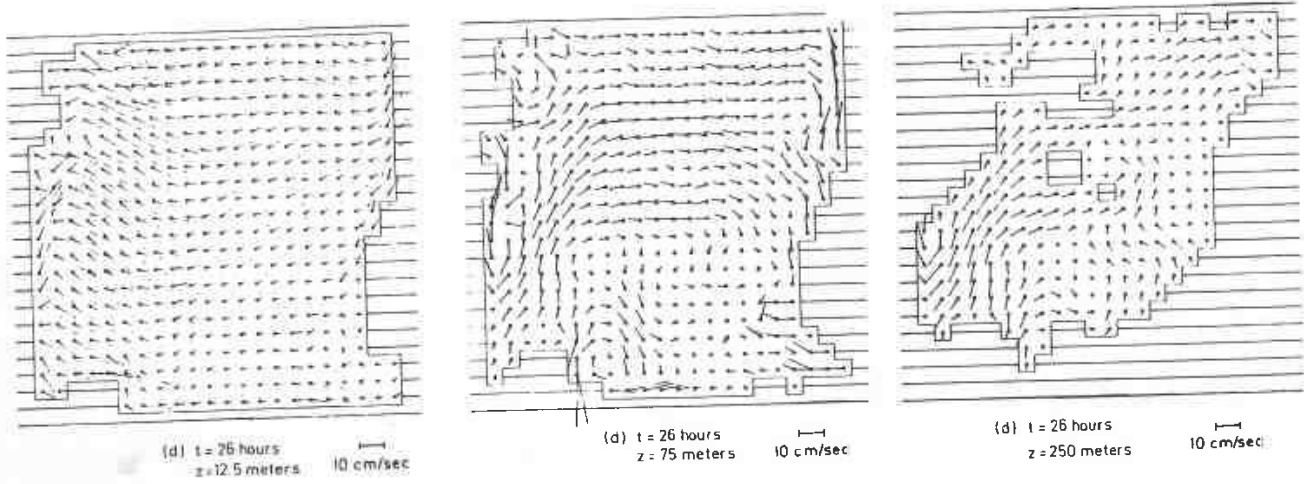

(d) $l=26$ hours $\quad 10 \mathrm{~cm} / \mathrm{sec}$
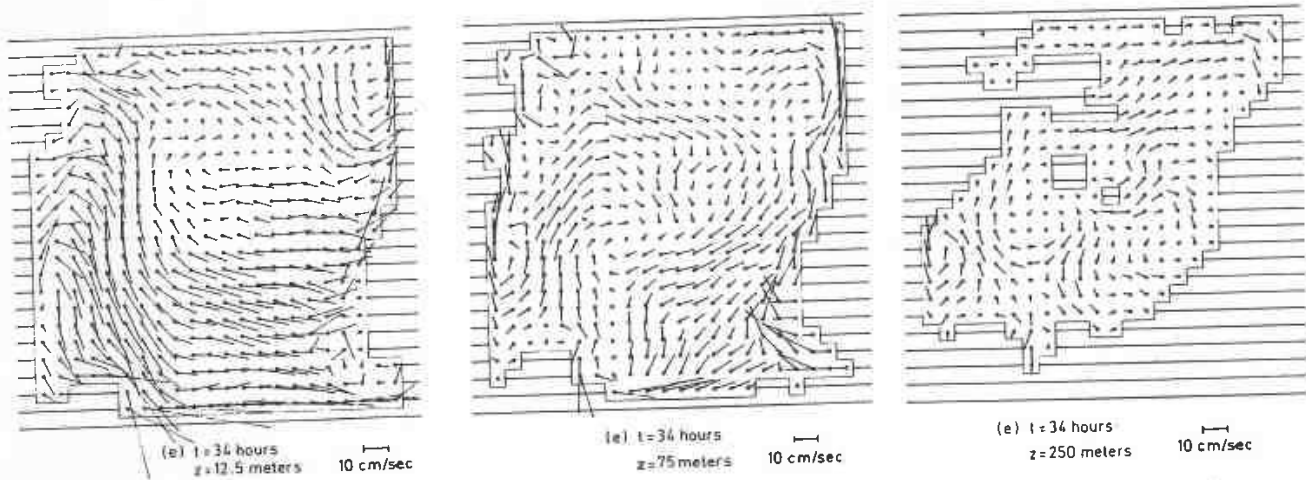

(e) $1=36$ hours

1.12 .5 meters $\quad 10 \mathrm{~cm} / \mathrm{sec}$

$x=75$ melers $\quad 10 \mathrm{~cm} / \mathrm{sec}$
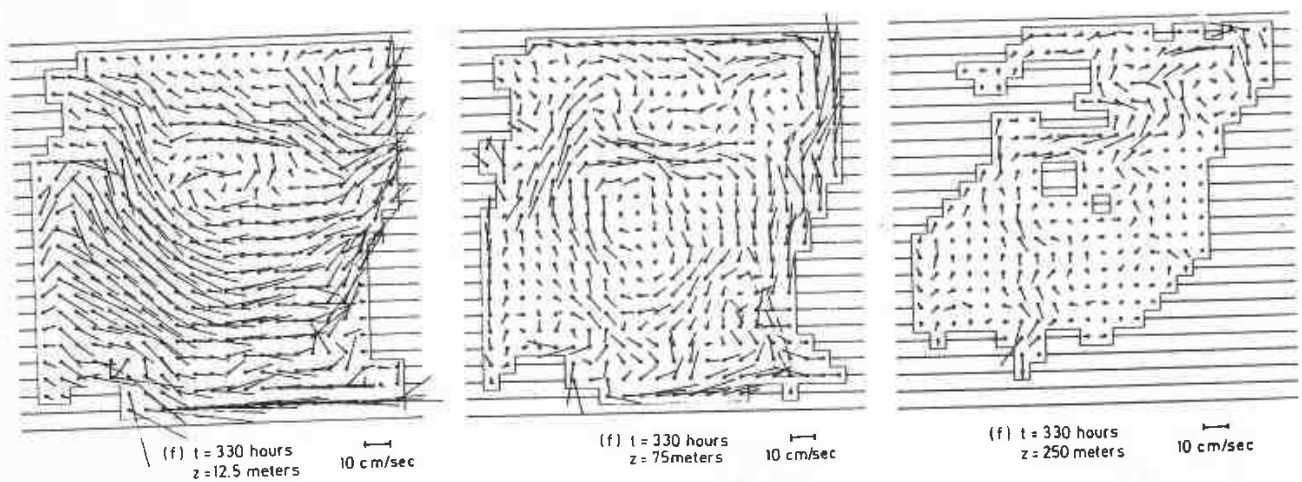

Figure 11. Horizontal velocity fields for times 26,34 and $330 \mathrm{~h}$, and depths $12 \cdot 5,75$ and $250 \mathrm{~m}$.

At $z=75 \mathrm{~m}$ the horizontal movement of water is characterized by a broad counterclockwise eddy in the middle of the sea, generated by the combined effect of the salinity distribution and the surface elevation. The horizontal pressure gradient is directed toward the centre of the basin in this upper part of the sea. The bottom currents at $z=250 \mathrm{~m}$ are mainly determined by bottom topography.

The effect of wind stress and vertical momentum exchange on the salinity distribution is shown to the right in Figs. 10 and 12. These figures show the space-time variations of the salinity profile at the middle west-east section of the flow field. An expected vertical mixing of salt and less salt water in the upper part of the ocean is 


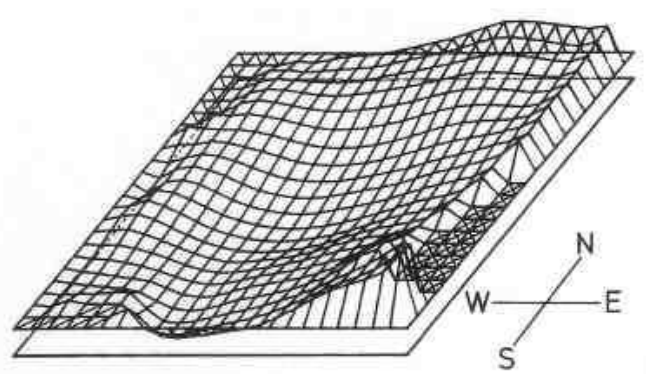

(d) $t=26$ hours

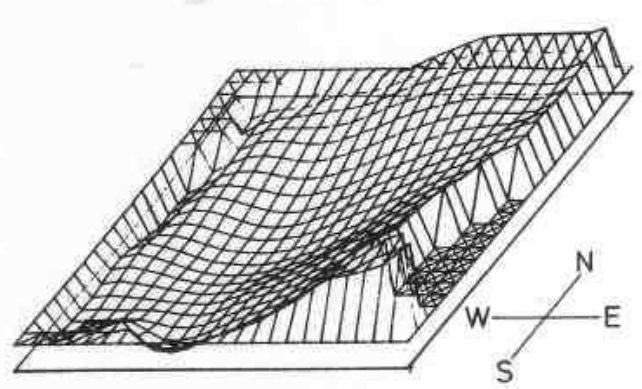

(e) $t=34$ hours

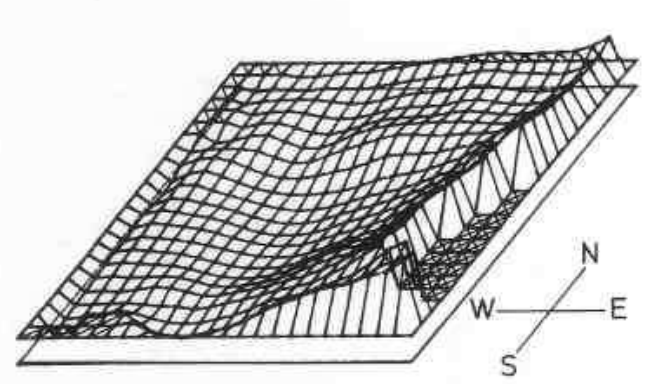

(f) $t=330$ hours

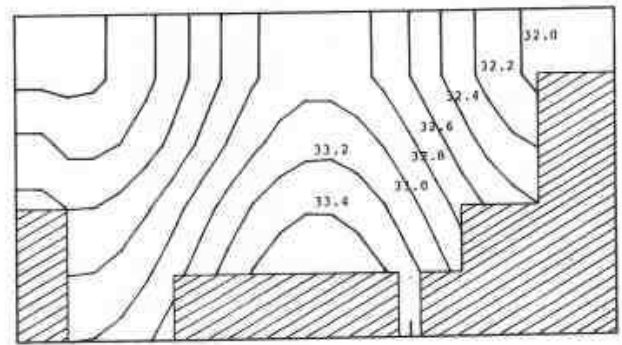

(d) $t=26$ hours

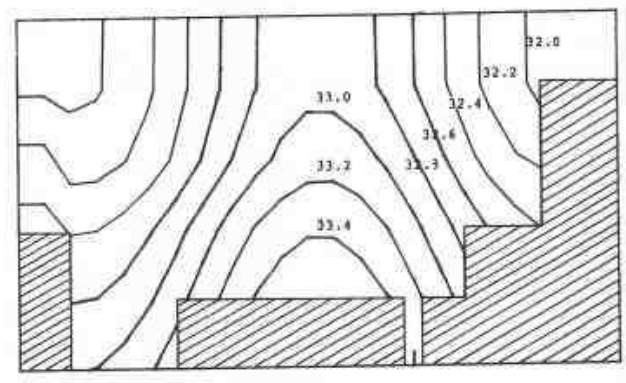

(e) $t=34$ hours

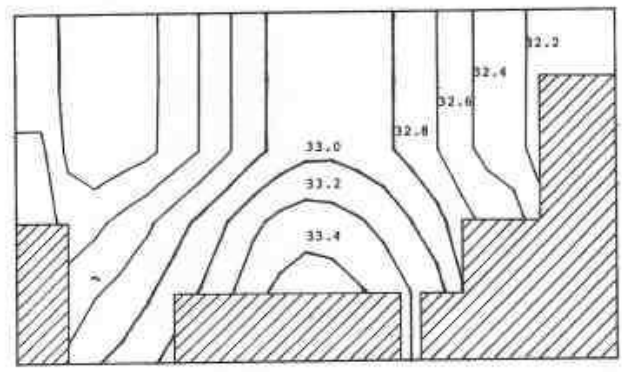

(f) $t=330$ hours

Figure 12. Left: Sea surface elevation plot for times 26,34 and $330 \mathrm{~h}$. The elevation vary between $20 \mathrm{~cm}$ below and $20 \mathrm{~cm}$ above the mean sea surface level. The land areas of Novaya Zemlya are marked by + , the northern parts of Norway by / and Spitsbergen by . Right: Salinity distributions for the east/west section located at $y=480 \mathrm{~km}$ for the times 26,34 and $330 \mathrm{~h}$. Note that each layer is represented by the same thickness in the figure.

obtained. Note the upwelling at the western boundary and the downwelling at the eastern boundary.

We shall not discuss the simulation results any further, but turn to the time efficiency of the splitting scheme for this computational problem. The efficiency results are tabulated in the Table.

The computer used is a medium size Nord-50 computer having a floating multiply execution time of approximately $5 \mu \mathrm{s}$. From the Table we observe that when using a slow mode time step of 80 minutes, which is shown to give the required accuracy, the 
split procedure is 16 times faster than the non-split procedure, and one month of real time simulation needs approximately 3.5 hours of computer time as compared to 56 hours computer time in the non-split case.

\section{Conclusion and summary}

A method for efficient numerical solution of the three-dimensional hydrodynamic equations of sea motion has been presented. The method is based on a splitting of the equations in a fast mode part and a slow mode part. The fast mode part includes the external gravity waves and the slow mode part represents the remaining part of the total model. A weak coupling term takes care of the interaction between the fast- and the slow-mode model parts. This algorithm is implemented by using an explicit leap frog type time scheme, except for the vertical eddy diffusion terms of the equations where a time implicit procedure is applied.

Two test problems are solved by simulation using this mode splitting procedure. First a two-dimensional problem is solved on a vertical grid to test the accuracy of the procedure. These results show no significant deviation from the non-split simulations when the time step of the slow mode part of the model is increased from 2 minutes (in the non-split case) to 80 minutes. Second, a fully three-dimensional problem on a realistic bottom topography is solved. Computer time measurements show that for slow mode time steps of $80 \mathrm{~min}$, the split model simulations are 16 times faster than the nonsplit simulations.

Hence, a very satisfactory increase in computational efficiency is obtained without a noticeable decrease in accuracy.

\section{Appendix}

\section{Choice of eddy viscosities}

When modelling ocean currents, the horizontal and vertical exchange of momentum comes into consideration. The horizontal eddy viscosity is assumed to be a constant.

The vertical eddy viscosity, $b$, is calculated, taking as a base the kinetic energy equation (Phillips 1966)

$$
b\left|v_{z}\right|^{2}+\frac{g}{\rho_{0}} \rho_{z} b_{\rho}-\epsilon=0
$$

The parameters in this equation are related as follows

$$
b=c_{0} l e \quad \epsilon=c_{1} e^{3} / l \quad \sigma=b_{\rho} / b
$$

where $l$ and $e^{2}$ are length scale and kinetic energy of the turbulent flow, respectively. Introducing (A 2) into (A 1) gives

$$
b\left|v_{z}\right|^{2}+\frac{g}{\rho_{0}} \rho_{z} b_{\rho}-\frac{c_{1}}{c_{0}} \frac{b^{3}}{l^{4}}=0
$$

Denoting $\frac{g}{\rho_{0}} \rho_{z}\left|\boldsymbol{v}_{z}\right|^{-2}$ as $\mathrm{Ri}$, and since $\frac{c_{1}}{c_{0}{ }^{3}}=1$, the expression for the eddy viscosity takes the form

$$
b=l^{2}\left|\boldsymbol{v}_{\mathbf{z}}\right|(1+\sigma \mathrm{Ri})^{\frac{1}{2}}
$$


In the case of non-stratified fluid this expression is simplified to the well-known Prandtl formula which relates eddy viscosity and length scale

$$
b=l^{2}\left|\boldsymbol{v}_{z}\right|
$$

Together with expressions (A 3) and (A 4) the empirical formula of Munch and Anderson (1948)

$$
b=b_{0}(1+\beta \mathrm{Ri})^{-\alpha}
$$

is used. Here $b_{0}$ is eddy viscosity in non-stratified fluid and $\beta \approx 10, \alpha \approx 0 \cdot 5$.

\section{ACKNOWLEDGMENT}

This work has been sponsored by The Royal Norwegian Council for Scientific and Industrial Research through the research program HAVBIOMODELLER (OCEAN BIO-MODELS).

\section{REFERENCES}

BALCHEN, J. G. (1979). Mathematical and numerical modeling of physical and biological processes in the Barents Sea. Statistical Ecology Series, Ed. G. P. Patil (Burtonville, Maryland, U.S.A.: International Cooperative Publishing House).

GourLAY, A. R. (1977). Splitting methods for time dependent partial differential equations, in The State of the Art in Numerical Analysis, ed. by D. Jacobs (Academic Press), pp. 757-796.

LEENDERTSE, J. J., ALEXANDER, R. C., and LIU, S. K. (1973). A three-dimensional model for estuaries and coastal seas: Vol. I, Principles of computation, R-1417-OWRR (The Rand Corporation, Santa Monica).

LEENDERTSE, J. J., and LIU, S. K. (1975). A three-dimensional model for estuaries and coastal seas: Vol. II, Aspects of computation, R-1764-OWRT (The Rand Corporation, Santa Monica).

LEENDERTSE, J. J., and LIU, S. K. (1977). A three-dimensional model for estuaries and coastal seas: Vol. IV, Turbulent energy computation, R-2787-ORWT (The Rand Corporation, Santa Monica).

LIU, S. K., and LeENDERTSE, J. J. (1978). Multidimensional numerical modeling of estuaries and coastal seas, Advances in Hydroscience, Vol. 11 (Academic Press), pp. 95-164.

Madala, R. V., and PiAcseK, S. A. (1977). A semi-implicit numerical model for baroclinic oceans, J. Comp. Physics, 23, pp. 167-178.

MunCH, W. H., and ANDERSEN, E. R. (1948). Note on the theory of thermocline, J. Mar. Research, 7, 276-295.

Peaceman, D. W., RACHFord, H. H., Jr. (1955). The numerical solution of parabolic and elliptic differential equations, SIAM J., 3, pp. 28-41.

Phillips, O. M. (1966). The dynamics of upper layer (University Press). 